\title{
A New Reconstruction of Variational Iteration Method and Its Application to Nonlinear Volterra Integrodifferential Equations
}

\author{
K. Maleknejad and M. Tamamgar \\ Department of Mathematics, Islamic Azad University, Karaj Branch, Karaj, Iran \\ Correspondence should be addressed to K. Maleknejad; maleknejad@iust.ac.ir \\ Received 1 February 2014; Accepted 8 April 2014; Published 28 April 2014 \\ Academic Editor: Morteza Khodabin
}

Copyright ( $) 2014$ K. Maleknejad and M. Tamamgar. This is an open access article distributed under the Creative Commons Attribution License, which permits unrestricted use, distribution, and reproduction in any medium, provided the original work is properly cited.

\begin{abstract}
We reconstruct the variational iteration method that we call, parametric iteration method (PIM). The purposed method was applied for solving nonlinear Volterra integrodifferential equations (NVIDEs). The solution process is illustrated by some examples. Comparisons are made between PIM and Adomian decomposition method (ADM). Also exact solution of the 3rd example is obtained. The results show the simplicity and efficiency of PIM. Also, the convergence of this method is studied in this work.
\end{abstract}

\section{Introduction}

It is well known that many events in scientific fields deal with integrodifferential equations. The nonlinear Volterra integrodifferential equations play a major role in many physical processes such as nanohydrodynamic [1], dropwise condensation [2], and biologic [3]. The various numerical methods exist for solving NVIDEs, for example, variational iteration method (VIM), Adomian decomposition method $[4,5]$, Chebyshev polynomials [6], and Bernstein's approximation [7]. First, Liao purposed homotopy analysis method $[8]$ and it is applied in many scientific problems $[9,10]$; then, VIM was purposed by $\mathrm{He}$ [11]. In this paper, we reconstruct the VIM that we call PIM. PIM was applied successfully for solving boundary value problems [12]. We consider nonlinear integrodifferential equations as follows:

$$
\begin{aligned}
& u^{(m)}(x)=f(x)+\lambda \int_{0}^{x} k(x, t) F(u(t)) d t, \quad a \leq x \leq b, \\
& u^{(m)}(x)=f(x)+\lambda \int_{0}^{x} k(x, t) F\left(u, u^{\prime}\right) d t, \quad a \leq x \leq b,
\end{aligned}
$$

and the initial value for both of the two equations is as follows:

$$
u^{(i)}\left(x_{0}\right)=y_{i}, \quad(i=0,1, \ldots, m-1) .
$$

In this work, the numerical solution of (1) and (2) is possible by PIM when $f, k, u$ are continuous and $F$ is continuous operator. Parametric iteration method provides solution for NVIDEs as a sequence of iterations. In this study, some examples are given and we solve them using parametric iteration method and compare the obtained results with $\mathrm{ADM}$ results. In all these cases, the present technique worked excellently, as it will be shown in this study.

\section{The Basic Idea of the Parametric Iteration Method}

In this section, we describe PIM for solving nonlinear Volterra integrodifferential equations. Then, the local convergence is discussed.

2.1. Parametric Iteration Method. The PIM provides the solution for (1) and (2) as a sequence of approximations. This method gives rapidly convergent successive approximations of the exact solution if such a solution exists; otherwise, approximations can be used for numerical purposes. We assume $L$ and $N$ are the linear and nonlinear operators on $C^{m}[a, b]$. To explain the basic idea of PIM, we consider (1) and (2) as follows:

$$
L(u)+N(u)=f(x),
$$


where $L$ with the property $L(g) \equiv 0$ when $g \equiv 0$ denotes the so-called auxiliary linear operator with respect to $u, N$ is a nonlinear continuous operator with respect to $u, f(x)$ is the known continuous function, and $u \in C^{m}[a, b]$. The basic essence of this method is to construct a family of iterative processes for (1) and (2) as [13]

$$
L\left[u_{k+1}(x)-u_{k}(x)\right]=h H(x) A\left[u_{k}(x)\right],
$$

with the initial conditions

$$
u^{(i)}\left(x_{0}\right)=y_{i}, \quad(i=0,1, \ldots, m-1),
$$

where

$$
\begin{aligned}
A\left[u_{k}(x)\right] & =L\left[\left(u_{k}(x)\right]+N\left[u_{k}(x)\right]-f(x)\right. \\
& =u_{k}^{(m)}-\lambda \int_{0}^{x} k(x, t) u_{k}^{n} d t-f(x),
\end{aligned}
$$

or

$$
\begin{aligned}
A\left[u_{k}(x)\right] & =L\left[\left(u_{k}(x)\right]+N\left[u_{k}(x)\right]-f(x)\right. \\
& =u_{k}^{(m)}-\lambda \int_{0}^{x} F\left(x, t, u_{k}, u_{k}^{\prime}\right) d t-f(x),
\end{aligned}
$$

and $u_{0}(x)$ is the initial guess which can be chosen arbitrarily, but the suitable selection is positively affect for the rate of convergence [13], or it can also be solved from its corresponding linear homogeneous equation $L\left[u_{0}(x)\right]=0$ or linear nonhomogeneous equation $L\left[u_{0}(x)\right]=f(x)$. The parameter $h \neq 0$ and function $H(x) \neq 0$ denote the so-called auxiliary parameter and auxiliary function. The selection of $h, H(x)$ was described in [13]. Also, we are free to choose the auxiliary linear operator $L$, the auxiliary parameter $h$, the auxiliary function $H(x)$, and the initial approximation $u_{0}(x)$. Therefore, if the successive approximations $u_{k}(x), k \geq 0$ are obtained by PIM in terms of the auxiliary parameter $h$, then exact solution may be given by $u(x)=\lim _{k \rightarrow \infty} u_{k}(x)$. According to [13], Let $V=\left\{u: u \in C^{m}[a, b]\right\}$ be the solution space and let $\left\{e_{j}(x): e_{j}(x) \in V, j=0,1, \ldots\right\}$ denote the set of base functions. Hence, we can represent the solution in the series $u(x)=\sum_{j=0}^{\infty} \alpha_{j} e_{j}(x)$, where $\alpha_{j}$ is a coefficient belonging to real numbers. As long as the set of base functions is determined, the auxiliary linear operator $L$, the initial approximation $u_{0}(x)$, and the auxiliary function $H(x)$ must be chosen in such a way that all solutions of the corresponding PIM equations (4) exist and it can be expressed by this set of base functions. Now, in order to avoid expensive computational works for solving (1) and (2) via PIM, it is straightforward to use the following set of base functions:

$$
\left\{(x-a)^{j} \mid j=0,1, \ldots\right\}
$$

that is,

$$
u(x)=\sum_{j=0}^{\infty} \alpha_{j}(x-a)^{j}
$$

where $\alpha_{j} \in R$ are unknown coefficients to be determined and $a$ is a constant belonging to real numbers. Now, we set the auxiliary operator $L$ as follows:

$$
L[u(x)]=u^{(m)}(x) .
$$

The initial guess is to form combination of $m$-terms of (9); that is,

$$
u_{0}(x)=\alpha_{0}+\alpha_{1}(x-a)+\alpha_{2}(x-a)^{2}+\cdots+\alpha_{j}(x-a)^{j} .
$$

According to (11) and the initial conditions (3) and with due attention to $L\left[u_{0}(x)\right]=f(x)$, the coefficients $\alpha_{0}, \alpha_{1}, \alpha_{2}, \ldots, \alpha_{m}$ will be determined. Also, we set $H(x)=1$. The selecting of $H(x)$ is arbitrary, but the suitable selection depends on the base functions for solution [13], and we use the PIM processes to compute the approximation solutions of (1) and (2).

2.2. The Valid Region of $h$. Assume that we gain a family of solution series in terms of the auxiliary parameter $h$ by means of PIM. We consider this solution as a function in terms of $h, x$; then, we derive (once or more) this function with respect to $x$ in $x=\beta$ that $\beta \in[a, b]$; that is, let $U=G(x, h)$ be the solution of (1) or (2); then, we set

$$
\Omega=\left.\frac{\partial^{i} G}{\partial x^{i}}\right|_{x=\beta}, \quad \beta \in[a, b], \quad(i=1,2, \ldots),
$$

therefore, $\Omega$ will be in terms of $h$; now we plot $\Omega$ curve, and according to these $h$ curves, it is easy to discover the valid region of $h$, which corresponds to the line segments nearly parallel to the horizontal axis. This region is called valid region of $h$ which we denote by $R_{h}$. We ensure that the solution series converge for any $h \in R_{h}$.

2.3. Analysis of Convergence of the Parametric Iteration Formula. In this section, we study the local convergence of approximate solution provided by PIM for solving (1). The convergence of approximate solution for (2) is similar to (1).

Initially, let $u^{(i)}(0)=0,(i=0,1, \ldots, m-1)$ and set $L[u(x)]=u^{(m)}(x)$; therefore, we have from (5) the following parametric iteration formula:

$$
\begin{aligned}
& u_{k+1}(x) \\
& =(1+h) u_{k}(x)-\frac{h}{(m-1) !} \\
& \quad \times \int_{0}^{x}(x-t)^{m-1}\left[g(t)+\lambda \int_{0}^{t} k(t, s) F\left(u_{k}(s)\right) d s\right] d t .
\end{aligned}
$$

The iterative formula (14) expressed by sequence makes a recurrence sequence $\left\{u_{k}(x)\right\}$. Obviously, the limit of the sequence will be the solution of (1) if the sequence is convergent. 
In order to prove that the sequence $\left\{u_{k}(x)\right\}$ is convergent, we construct a series:

$$
u_{0}(x)+\left[u_{1}(x)-u_{0}(x)\right]+\cdots+\left[u_{k}(x)-u_{k-1}(x)\right]+\cdots .
$$

Noticing that

$$
\begin{aligned}
s_{k+1}= & u_{0}(x)+\left[u_{1}(x)-u_{0}(x)\right]+\cdots \\
& +\left[u_{k}(x)-u_{k-1}(x)\right]=u_{k}(x),
\end{aligned}
$$

the sequence $\left\{u_{k}(x)\right\}$ will be convergent if the series is convergent.

Theorem 1. If $F(u(t))$ is Lipschitz-continuous in $[a, b]$ and $g(x) \in C[a, b]$ and $|\lambda| \leq 1 / M N$ where $M, N$ are positive real numbers, then the series of (15) is convergent; that is, the sequence $\left\{u_{k}(x)\right\}$ is convergent for $x \in[a, b]$.

Proof. According to (14), note that

$$
\begin{aligned}
& \left|u_{1}(x)-u_{0}(x)\right| \\
& =\mid h\left[u_{0}(x)-\frac{1}{(m-1) !}\right. \\
& \left.\times \int_{0}^{x}(x-t)^{m-1}\left[g(t)+\lambda \int_{0}^{t} k(t, s) F\left(u_{0}(s)\right) d s\right] d t\right] \mid \\
& \leq|h|\left[L_{1}+\frac{1}{(m-1) !}\left(L_{2} L_{3} b+\frac{L_{2} L_{4}}{2 N} b^{2}\right)\right]=|h| r,
\end{aligned}
$$

where

$$
\begin{gathered}
r:=\left(L_{1}+\frac{1}{(m-1) !}\right)\left(L_{2} L_{3} b+\frac{L_{2} L_{4}}{2 N} b^{2}\right), \\
M=\max _{a \leq t \leq x \leq b}|k(x, t)|, \quad L_{1}=\max _{a \leq t \leq x \leq b}\left|u_{0}(t)\right|, \\
L_{2}=\max _{a \leq t \leq x \leq b}\left|(x-t)^{m-1}\right|, \quad L_{3}=\max _{a \leq t \leq x \leq b}|g(t)|, \\
L_{4}=\max _{a \leq t \leq x \leq b}\left|F\left(u_{0}(t)\right)\right| .
\end{gathered}
$$

From (14) and (17) and the assumption that $\mid F\left(u_{k+1}\right)-$ $F\left(u_{k}\right)|\leq N| u_{k+1}-u_{k} \mid$ where $N$ denotes the Lipschitz constant of $F(u(t))$, it follows that

$$
\begin{aligned}
& \quad\left|u_{2}-u_{1}\right| \\
& =\mid\left[(1+h)\left(u_{1}-u_{0}\right)-\frac{h}{(m-1) !}\right. \\
& \left.\quad \times \int_{0}^{x}(x-t)^{m-1}\left[\lambda \int_{0}^{t} k(t, s)\left(F\left(u_{1}\right)-F\left(u_{0}\right)\right) d s\right] d t\right] \mid \\
& \leq|| 1+h|| u_{1}-u_{0} \mid+\frac{|h|}{(m-1) !}
\end{aligned}
$$

$$
\begin{aligned}
& \times \int_{0}^{x}(x-t)^{(m-1)}\left[\lambda N \int_{0}^{t} k(t, s)\left|u_{1}-u_{0}\right| d s\right] d t \mid \\
\leq & |h| r\left[|1+h|+\frac{L_{2}|h|}{2(m-1) !} b^{2}\right] \\
= & |h| r \sum_{n=0}^{1}\left(\begin{array}{l}
1 \\
n
\end{array}\right)|1+h|^{1-n}\left(\frac{L_{2}|h|}{2(m-1) !} b^{2}\right)^{n}
\end{aligned}
$$$$
\left|u_{3}-u_{2}\right|
$$$$
=\mid\left[(1+h)\left(u_{2}-u_{1}\right)-\frac{h}{(m-1) !}\right.
$$$$
\left.\times \int_{0}^{x}(x-t)^{m-1}\left[\lambda \int_{0}^{t} k(t, s)\left(F\left(u_{2}\right)-F\left(u_{1}\right)\right) d s\right] d t\right] \mid
$$$$
\leq|| 1+h|| u_{2}-u_{1} \mid+\frac{|h|}{(m-1) !}
$$$$
\times \int_{0}^{x}(x-t)^{(m-1)}\left[\lambda N \int_{0}^{t} k(t, s)\left|u_{2}-u_{1}\right| d s\right] d t \mid
$$$$
\leq|h| r\left[|1+h|^{2}+\frac{2 L_{2}|h||1+h|}{2(m-1) !} b^{2}+\frac{L_{2}{ }^{2}|h|^{2}}{(2(m-1) !)^{2}} b^{4}\right]
$$$$
=|h| r \sum_{n=0}^{2}\left(\begin{array}{l}
2 \\
n
\end{array}\right)|1+h|^{1-n}\left(\frac{L_{2}|h|}{2(m-1) !} b^{2}\right)^{n}
$$$$
\vdots
$$

$$
\left|u_{k+1}-u_{k}\right| \leq(|h| r) \sum_{n=0}^{k}\left(\begin{array}{l}
k \\
n
\end{array}\right)|1+h|^{k-n}\left(\frac{L_{2}|h| b^{2}}{2(m-1) !}\right)^{n} \text {. }
$$

In view of (21), the convergence of the series (15) can be concluded for the solution domain $x>0$ and $|1+h|<1$ with the help of some mathematical software. Therefore, the series of (15) is absolute convergence; that is, the sequence $\left\{u_{k}(x)\right\}$ is convergent for $x \in[a, b]$.

\section{Illustrative Examples}

Now, we use PIM to solve two examples of the kind of (1) and (2) and compare the obtained results with ADM [5] to show the efficiency of PIM.

Example 1. The first example is a nonlinear Volterra integrodifferential equation of the second kind as follows:

$$
\begin{gathered}
u^{\prime}(x)=-1+\int_{0}^{x} u^{2}(t) d t, \quad 0 \leq x \leq 1 \\
u(0)=0 .
\end{gathered}
$$




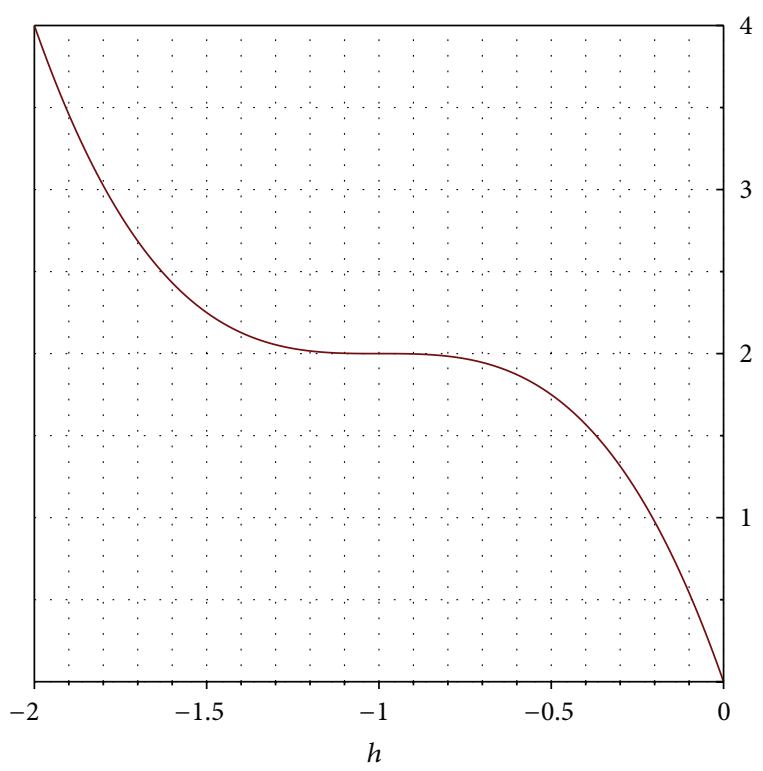

Figure 1: The valid region of $h$. Example 1 shows $-1.2 \leq h \leq-0.8$.

According to PIM proceeding, we define

$$
\begin{gathered}
L[u(x)]=u^{\prime}(x), \\
N[u(x)]=\int_{0}^{x} u^{2}(t) d t, \quad f(x)=-1 .
\end{gathered}
$$

Then, we have from (10) that $u_{0}(x)=\alpha_{0}+\alpha_{1} x$. Using initial condition and $L\left[u_{0}(x)\right]=f(x)$ gives us, that $\alpha_{0}=0, \alpha_{1}=$ -1 that is, $u_{0}(x)=-x$. Now, If we set $H(x)=1$, then we obtain from (5) and (8) that

$$
u_{k+1}^{\prime}(x)-u_{k}^{\prime}(x)=h\left(u_{k}^{\prime}(x)-\int_{0}^{x} u_{k}^{2}(t) d t+1\right) .
$$

Thus we integrate from both sides of (25); then the PIM equations are as follows:

$$
\begin{array}{r}
u_{k+1}(x)=u_{k}(x)+h \int_{0}^{x}\left[\left(u_{k}^{\prime}(t)-\int_{0}^{t} u_{k}^{2}(s) d s+1\right)\right] d x, \\
(k=0,1, \ldots) .
\end{array}
$$

Some of the iterations obtained from (25) are as follows and the other iterations have also been calculated by Maple 13:

$$
\begin{aligned}
u_{1}(x)=-x & +h\left(-\frac{1}{12} x^{4}\right), \\
u_{2}(x)=- & x-\frac{1}{12} h x^{4} \\
& +h\left(-\frac{1}{12960} h^{2} x^{10}-\frac{1}{252} h x^{7}+\frac{1}{4}\left(-\frac{1}{3} h-\frac{1}{3}\right) x^{4}\right) .
\end{aligned}
$$

The obtained result for the 3rd iteration was shown in Table 1; also the valid region of $h$ that is $R_{h}$ was presented in Figure 1.
TABLE 1: The results of Example 1 for $u_{3}(x)$ with $h=-1$.

\begin{tabular}{lcc}
\hline$x$ & ADM & PIM \\
\hline 0.0000 & 0.000000000 & 0.0000000 \\
0.0938 & -0.0937935 & -0.0937935 \\
0.2188 & -0.2186090 & -0.2186091 \\
0.3125 & -0.3117060 & -0.3117064 \\
0.4062 & -0.4039390 & -0.4039385 \\
0.5000 & -0.4948230 & -0.4948225 \\
0.6250 & -0.6124310 & -0.6124306 \\
0.7188 & -0.6969410 & -0.6969414 \\
0.8125 & -0.7770900 & -0.7770900 \\
0.9062 & -0.8519340 & -08519338 \\
1.0000 & -0.9204760 & -0.9204746 \\
\hline
\end{tabular}

Example 2. We consider the nonlinear Volterra integrodifferential equation of the second kind as follows:

$$
u^{\prime}(x)=1+\int_{0}^{x} u(t) u^{\prime}(t) d t, \quad 0 \leq x \leq 1,
$$

$$
u(0)=0 .
$$

According to PIM procedure we set

$$
\begin{gathered}
L[u(x)]=u^{\prime}(x), \\
N[u(x)]=\int_{0}^{x} u(t) u^{\prime}(t) d t, \quad f(x)=1 .
\end{gathered}
$$

Then, we have from (10) $u_{0}(x)=\alpha_{0}+\alpha_{1} x$, and (29) gives us that $\alpha_{0}=0, \alpha_{1}=1$; that is, $u_{0}(x)=x$. Now similar to Example 1, the iteration scheme is as follows:

$$
\begin{array}{r}
u_{k+1}(x)=u_{k}(x) \\
+h \int_{0}^{x}\left[\left(u_{k}^{\prime}(t)-\int_{0}^{t} u_{k}(s) u_{k}^{\prime}(s) d s-1\right)\right] d x \\
\quad(k=0,1, \ldots) .
\end{array}
$$

Some of the iterations obtained from (30) are as follows:

$$
\begin{aligned}
u_{1}(x)= & x-\frac{1}{6} h x^{3} \\
u_{2}(x)= & x-\frac{1}{6} h x^{3} \\
& +h\left(-\frac{1}{504} h^{2} x^{7}+\frac{1}{30} h x^{5}+\frac{1}{3}\left(-\frac{1}{2} h-\frac{1}{2}\right) x^{3}\right) . \\
& \vdots
\end{aligned}
$$

The results of Example 2 are available in Table 2. $R_{h}$ for (27) is presented in Figure 2. 
TABLE 2: The results of Example 2 for $u_{3}(x)$ with $h=-1$.

\begin{tabular}{lcc}
\hline$x$ & ADM & PIM \\
\hline 0.0000 & 0.0000000 & 0.0000000 \\
0.0938 & 0.0938065 & 0.0939377 \\
0.2188 & 0.2189910 & 0.2205626 \\
0.3125 & 0.3132980 & 0.3176876 \\
0.4062 & 0.4084910 & 0.4177517 \\
0.5000 & 0.5053030 & 0.5219304 \\
0.6250 & 0.6381770 & 0.6691415 \\
0.7188 & 0.7422990 & 0.7878390 \\
0.8125 & 0.8518530 & 0.9155129 \\
0.9062 & 0.9691440 & 1.0546355 \\
1.0000 & 1.0973700 & 1.2083812 \\
\hline
\end{tabular}

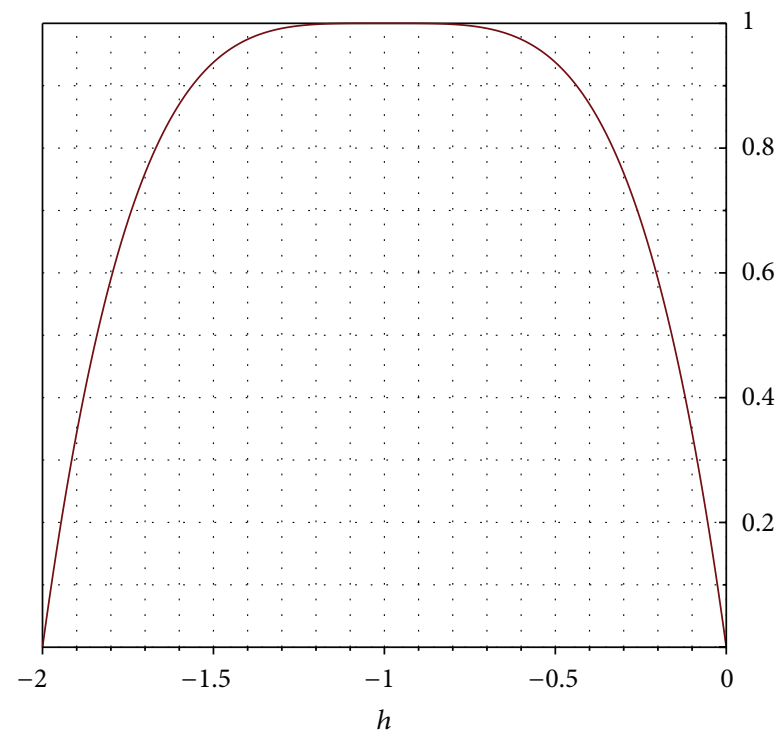

FIGURE 2: The valid region of $h$. Example 2 shows $-1.2 \leq h \leq-0.8$.

Example 3. We consider the nonlinear Volterra integrodifferential equation of the second kind as follows:

$$
\begin{gathered}
u^{\prime}(x)=1-\frac{x}{2}+\frac{x e^{-x^{2}}}{2}+\int_{0}^{x} x t e^{-u^{2}(t)} d t, \quad 0 \leq x \leq 1, \\
u(0)=0 .
\end{gathered}
$$

Similar to Examples 1 and 2

$$
\begin{gathered}
L[u(x)]=u^{\prime}(x), \\
N[u(x)]=\int_{0}^{x} x t e^{-u^{2}(t)} d t, \quad f(x)=1-\frac{x}{2}+\frac{x e^{-x^{2}}}{2} .
\end{gathered}
$$

Now, we consider the initial solution as $u_{0}(x)=\alpha_{0}+$ $\alpha_{1} x$, and (33) and (34) give us that $\alpha_{0}=0, \alpha_{1}=1$; that is, $u_{0}(x)=x$. According to PIM iterative formula for this example we have

$$
\begin{aligned}
u_{k+1}(x)=(1+h) u_{k}(x) & \\
+ & h\left[\frac{1}{4}+x-\frac{1}{4} x^{2}-\frac{1}{4} e^{x^{2}}\right. \\
& \left.+\left(\int_{0}^{x} \int_{0}^{t} t s e^{-u_{k}^{2}(s)} d s\right)\right] d t \\
& (k=0,1, \ldots) .
\end{aligned}
$$

Starting with $k=0$ and initial solution that is $u_{0}(x)=$ $x$, the exact solution of this example, which is $u(x)=x$, is achieved. The valid value of parameter for (36) is $h=-1$.

\section{Results}

In this section, we present the results of Examples 1 and 2 in two tables and plot the $h$-curve to determine $R_{h}$. All the computations have been done with Maple 13.

\section{Conclusion}

In this paper, we reconstruct the VIM that we call parametric iteration method, and PIM was applied to solve the nonlinear Volterra integrodifferential equations. In order to illustrate the method, we solve three examples. PIM results compared to $\mathrm{ADM}$ show that the former is easier in practice and more accurate for NVIDEs. For the 3rd example exact solution was achieved. Further, the convergence of PIM for solving NVIDEs in the valid region of $h\left(R_{h}\right)$ was presented. Additionally, if we increase the number of iterations by PIM scheme, it seems that the results will have more accuracy in solutions.

\section{Conflict of Interests}

The authors declare that there is no conflict of interests regarding the publication of this paper.

\section{References}

[1] L. Xu, J. H. He, and Y. Liu, "Electro spun nonporous spheres with Chinese drug," The International Journal of Nonlinear Sciences and Numerical Simulation, vol. 8, no. 2, pp. 199-202, 2007.

[2] F. Z. Sun, M. Gao, S. H. Lei et al., "The fractal dimension of the fractal model of dropwise condensation and its experimental study," International Journal of Nonlinear Sciences and Numerical Simulation, vol. 8, no. 2, pp. 211-222, 2007.

[3] A. J. Jerri, , Introduction to Integral Equations with Applications, Marcel Dekker, New Yourk, NY, USA, 1985.

[4] J. Saberi-Nadjafi and M. Tamamgar, "The variational iteration method: a highly promising method for solving the system of integro-differential equations," Computers \& Mathematics with Applications, vol. 56, no. 2, pp. 346-351, 2008.

[5] S. M. El-Sayed and M. R. Abdel-Aziz, "A comparison of Adomian's decomposition method and wavelet-Galerkin method for solving integro-differential equations," Applied Mathematics \& Computation, vol. 136, no. 1, pp. 151-159, 2003. 
[6] K. Maleknejad, S. Sohrabi, and Y. Rostami, "Numerical solution of nonlinear Volterra integral equations of the second kind by using Chebyshev polynomials," Applied Mathematics \& Computation, vol. 188, no. 1, pp. 123-128, 2007.

[7] K. Maleknejad, E. Hashemizadeh, and R. Ezzati, "A new approach to the numerical solution of Volterra integral equations by using Bernstein's approximation," Communications in Nonlinear Science and Numerical Simulation, vol. 16, no. 2, pp. 647-655, 2011.

[8] J. Sh. Liao, The proposed homotopy analysis technique for the solution of nonlinear problems [Ph.D. thesis], Shanghai Jiao Tong University, 1992.

[9] S. Abbasbandy, M. Ashtiani, and E. Babolian, "Analytic solution of the Sharma-Tasso-Olver equation by homotopy analysis method," Zeitschrift für Naturforschung Section A, vol. 65, no. 4, pp. 285-290, 2010.

[10] S. Abbasbandy and A. Shirzadi, "Homotopy analysis method for multiple solutions of the fractional Sturm-Liouville problems," Numerical Algorithms, vol. 54, no. 4, pp. 521-532, 2010.

[11] J.-H. He, "Variational iteration method-some recent results and new interpretations," Journal of Computational and Applied Mathematics, vol. 207, no. 1, pp. 3-17, 2007.

[12] A. Ghorbani, M. Gachpazan, and J. Saberi-Nadjafi, "A modified parametric iteration method for solving nonlinear second order BVPs," Computational and Applied Mathematics, vol. 30, no. 3, pp. 499-515, 2011.

[13] S. Liao, Beyond Perturbation: Introduction to the Homotopy Analysis Method, vol. 2 of CRC Series: Modern Mechanics and Mathematics, Chapman \& Hall/CRC, Boca Raton, Fla, USA, 2004. 


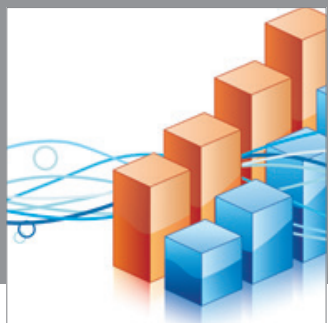

Advances in

Operations Research

mansans

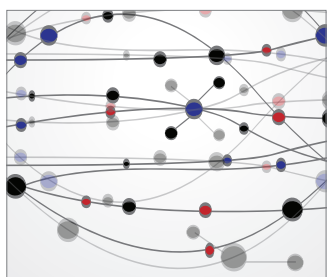

The Scientific World Journal
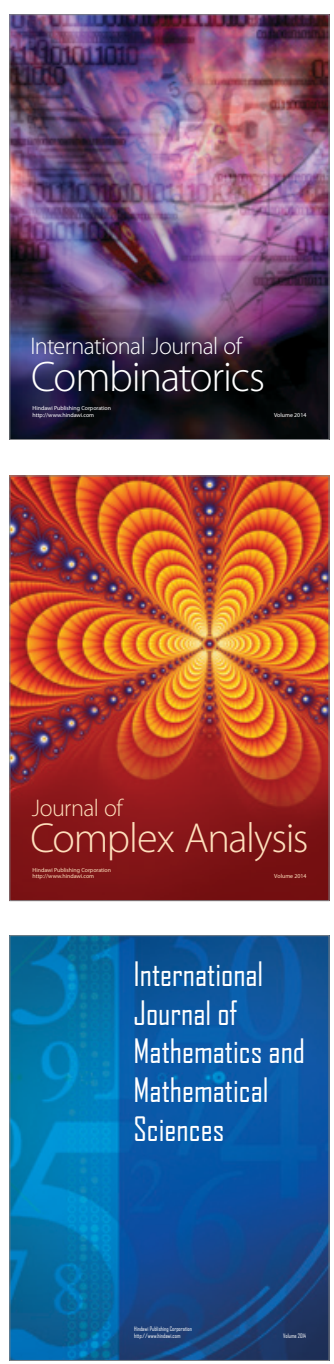
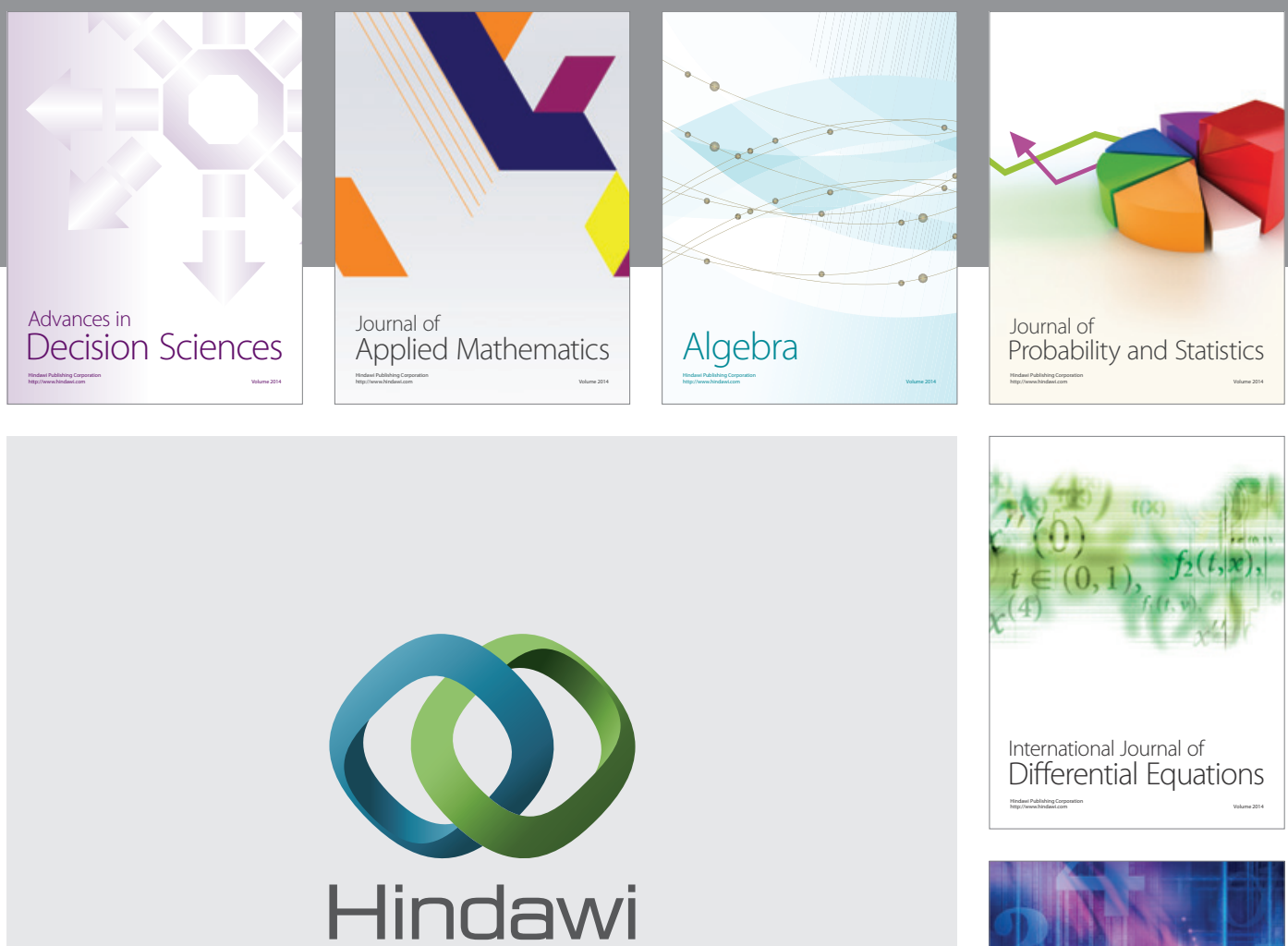

Submit your manuscripts at http://www.hindawi.com
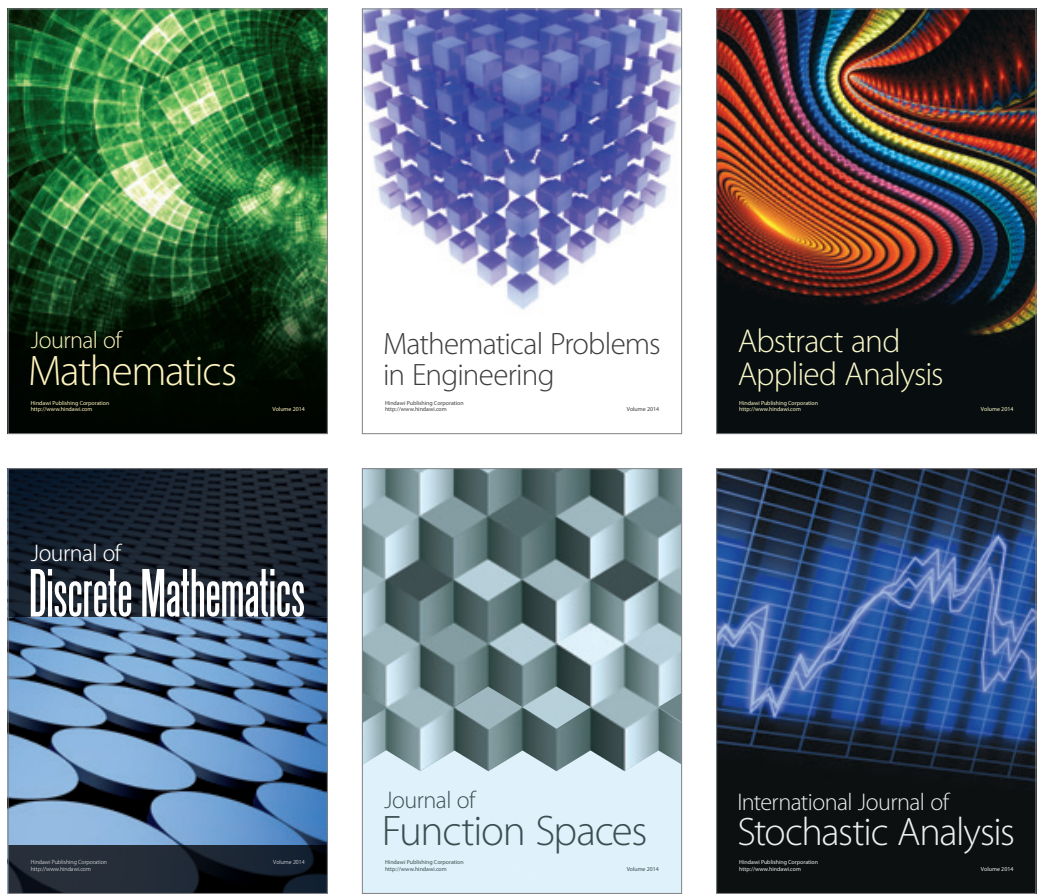

Journal of

Function Spaces

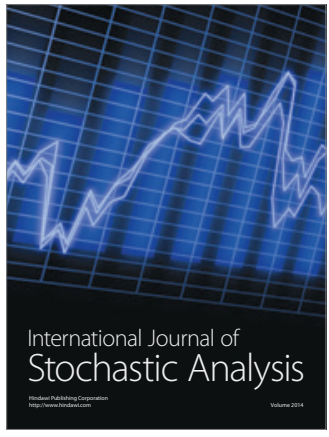

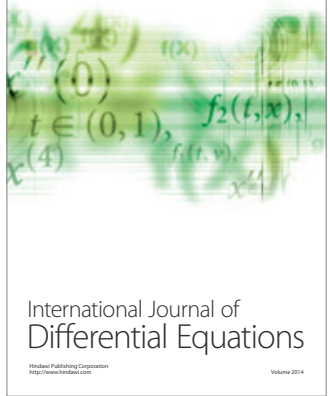
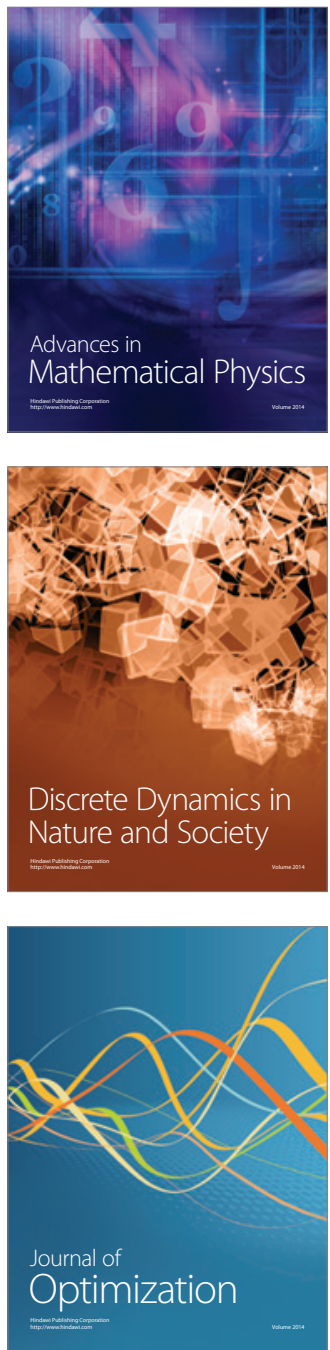